Plumbaginaceae (partim)-Capparaceae. Real Jardín Botánico. CSIC. Madrid.

MOTA, J.F., A.J. SOLA, A. AGUILERA, M.I. CERRILLO \& E. DANA -2003-. The Mediterranean Island of Alborán: A review of its flora and vegetation. Fitosociologia 39 (1): 15-21. NAVARRO, T. -1995- Revisión del género Teucrium L. sección polium (Mill.) Schreb. (Lamiaceae) en la Península Ibérica. Acta Botanica Malacitana 20: 173-265.

SAGREDO, R. -1987- Flora de Almería. Plantas vasculares de la provincia. I.E.A. Diputación de Almería.

TUTIN, T.G. et al. [eds.] -1964/1980- Flora Europaea. vols I-V. Cambridge University Press.
VALDÉS BERMEJO, E. -1993- Reseda L. en Castroviejo, S. et al. (Eds.) Flora Iberica III: Cruciferae-Monotropaceae. Real Jardín Botánico. CSIC. Madrid.

VALDÉS, B, S. TALAVERA y F. GALIANO (eds.) -1987- Flora vascular de Andalucía Occidental. Vols. I-III. Ed. Ketres. Barcelona.

Aceptado para su publicación en octubre de 2003

Dirección de los autores. Dpto. Biología Vegetal y Ecología. Univ. de Almería . 04120 Almería.

\title{
90. NOVEDADES COROLÓGICAS PARA LA COMARCA DEL ANDÉVALO (PROVINCIA DE HUELVA, SO DE ESPAÑA). IV.
}

\author{
Consuelo SANTA-BÁRBARA, Benito VALDÉS y Francisco José PINA
}

New floristic records for Andevalo area (Huelva Province, SW Spain). IV.

Palabras clave. Monocotiledóneas, corología, Andévalo, Andalucía, SO de España, florística.

Key words. Monocotyledons, chorology, Andévalo, Andalucia, SW Spain, floristic.

Este trabajo completa una serie de artículos sobre novedades corológicas de la comarca del Andévalo (Santa-Bárbara, 1997a, Santa-Bárbara, 1997b, Santa-Bárbara et al., 2003). Esta comarca ocupa el centro de la provincia de Huelva, desde la frontera con Portugal por el oeste hasta la provincia de Sevilla por el este. Su límite norte lo constituye la comarca serrana de Aracena y por el sur la limitan las comarcas más meridionales de la Campiña y Litoral onubense.

Se trata de Monocotiledóneas, que se conocían de otras comarcas de Andalucía Occidental pero no del Andévalo. Se indica para cada una de ellas las localidades en las que se han recolectado y las áreas en que eran conocidas en la mitad occidental de Andalucía, según Flora Vascular de Andalucía Occidental 
(Valdés et al., 1987), siguiendo para ello la división en comarcas naturales de esta flora. Se incluyen además comentarios sobre los hábitats en los que se han recolectado en el área de estudio.

Los taxones se disponen en orden taxonómico, siguiendo el adoptado en la citada Flora Vascular.

\section{Alisma lanceolatum With.}

HUELVA. Ayamonte: Arroyo Pedraza, PB4725, 2.7.96, Santa-Bárbara (SEV 150738); ídem, Prado de la Noria, PB4026, 25.4.97, SantaBárbara \& Valdés (SEV 150739). Villablanca: Entre Villablanca y San Silvestre de Guzmán, PB4631, 21.5.94, Santa-Bárbara (SEV 150740).

Frecuente en las comarcas de los Pedroches, Campiña de Huelva, Condado-Aljarafe, Vega, Litoral, Marisma y Algeciras. Ha sido citada recientemente para la comarca de la Sierra Norte de Sevilla (Pérez Latorre et al., 2002: 204). Rara en la comarca del Andévalo, donde sólo se ha localizado en su extremo suroccidental. Se encuentra en bordes de cursos de agua o en lugares encharcados.

\section{Potamogeton fluitans Roth}

Potamogeton nodosus Poir.

HUELVA. Paymogo: Río Chanza y alrededores, PB4080 12.8.95, Santa-Bárbara (SEV 150765). Zalamea la Real: Rivera de Cachán, QB16, 13.7.90, Santa-Bárbara (SEV 150766).

Esta especie, con distribución limitada en Andalucía Occidental a las comarcas de Alcores, Campiña Alta y Subbética, ha sido localizada en el Andévalo, siendo ésta la primera cita para la comarca. Se encuentra en zonas remansadas de cursos de agua.

\section{Potamogeton pusillus L.}

HUELVA. Ayamonte: Carril hacia el Guadiana, PB4535, 22.5.96, Santa-Bárbara (SEV 150770). Sanlúcar de Guadiana: Entre San Silvestre de Guzmán y Sanlúcar de Guadiana, PB4343, 11.6.94, Santa-Bárbara (SEV 150771); ídem, Entre San Silvestre de Guzmán y Sanlúcar de Guadiana, PB4045, 11.6.94, Santa-Bárbara (SEV 150772). Villablanca: Carril hacia el Guadiana, arroyo Grande, PB4432, 22.5.96, Santa-Bárbara (SEV 150773).
Se conocía de las comarcas de los Pedroches, Sierra Norte y Subbética. Fernández Galiano \& Cabezudo (1976: 161) citan este taxón para el Litoral de Huelva, en charcas y lagunas de Doñana. Recientemente se ha localizado en el Andévalo, sólo en el suroeste de la comarca, en charcas y arroyos.

Zannichellia obtusifolia Talavera, García-Mur. \& Smit HUELVA. Villanueva de los Castillejos: Camino a Casa del Rinconcillo, PB5044, 10.4.96, Santa-Bárbara (SEV 150774).

Especie frecuente en las comarcas de los Pedroches, Litoral, Marisma, Campiña Baja y Algeciras. Muy rara en el Andévalo, donde se cita por primera vez. Se ha localizado en un único enclave, en una charca de aguas someras.

\section{Lemna minor $\mathrm{L}$.}

HUELVA. Sanlúcar de Guadiana: Entre San Silvestre de Guzmán y Sanlúcar, PB44, 30.9.97, Santa-Bárbara (SEV 150799).

Distribuida originalmente en las comarcas de Sierra Norte, Aracena, Litoral y Algeciras. Es rara en la comarca del Andévalo, donde se cita por primera vez. Se ha localizado en las orillas remansadas de un pequeño embalse.

\section{Juncus acutus L. var. acutus}

HUELVA. Ayamonte: Río Guadiana, PB3829, 28.2.96, Santa-Bárbara (SEV 150827); ídem, Arroyo Pedraza, PB4725, 2.7.96, Santa-Bárbara (SEV 150828). El Almendro: Barranco de la Murtilla, PB4458, 29.5.96, Santa-Bárbara (SEV 150829). El Campillo: Alrededores de la represa de Gossan, QB1279, 29.3.90, Santa Bárbara (SEV 150830). Puebla de Guzmán: Carretera a E1 Almendro, PB5462, 12.6.93, Santa-Bárbara (SEV 150831); ídem, a $5 \mathrm{~km}$ de Puebla de Guzmán hacia El Almendro, PB5559, 11.6.94, Santa-Bárbara (SEV 150832). Villablanca: Carril hacia el Guadiana, arroyo Grande, PB4432, 22.5.96, SantaBárbara (SEV 150833). Villanueva de los Castillejos: A $3 \mathrm{Km}$ del pueblo viniendo de El Granado, 25.2.94, Santa-Bárbara \& Valdés (SEV 150834).

Ampliamente distribuida en Andalucía Occidental, no había sido citada con anterioridad en la comarca del Andévalo, donde se encuentra en bordes de cursos de agua y charcas. Es más corriente en aguas salobres. 


\section{Juncus inflexus L. var. inflexus}

HUELVA. El Campillo: Arroyo que desemboca en el Zumajo, QB1273, Santa-Bárbara (SEV 150837). El Granado: Puerto de la Laja, PB3354, 26.4.97, Santa-Bárbara \& Valdés (SEV 150835). Paymogo: Río Chanza y alrededores, PB4080 12.8.95, Santa-Bárbara (SEV 150838). Villanueva de las Cruces: A un $\mathrm{Km}$ de Villanueva de las Cruces, arroyo Cascabelero, PB7465, 15.5.96, Santa-Bárbara (SEV 150839).

Este taxón presenta una distribución que cubre las comarcas de Zújar, Pedroches, Sierra Norte, Aracena, Litoral, Campiña Baja de Cádiz, Campiña Alta de Cádiz, Subbética de Cádiz, Grazalema y Algeciras. En el Andévalo ha sido localizada en bordes de cursos de agua, en general en zonas nitrificadas.

\section{Juncus foliosus Desf.}

HUELVA. El Cerro del Andévalo: Entre Cabezas Rubias y Calañas, a un Km del cruce a Montes de San Benito, PB7476, 24.3.96, SantaBárbara (SEV 150937). Entre El Granado y Villanueva de los Catillejos, 11.3.97, Cabezudo \& Silvestre (SEV 27311). El Granado: Carretera al Puerto de la Laja, PB3354, 15.5.93, Santa-Bárbara (SEV 150938). Puebla de Guzmán: Carril de Puebla de Guzmán a la ermita Virgen de la Peña, PB5863, 24.3.96, Santa-Bárbara (SEV 150939). Santa Bárbara de Casa: Entre Santa Bárbara y Paymogo, barranco de Monteviejo, PB5482, 19.4.94, SantaBárbara (SEV 150940). Valverde del Camino: A 2 Km de La Florida, QB0454, 5.6.96, Santa-Bárbara (SEV 150941); ídem, Barranco de la Melera Chica, QB0354, 26.6.96, Santa-Bárbara (SEV 150942). Villanueva de los Castillejos: Entre Villanueva de los Castillejos y San Bartolomé, eucaliptal joven, PB6346, 10.4.96, Santa-Bárbara (SEV 150944). SEVILLA. El Madroño: Arroyo del Porzuño, QB2474, 7.4.93, Santa-Bárbara (SEV 150945).

Especie que cubre las comarcas de Aracena, Litoral y partes bajas de Algeciras. Se cita por primera vez para la comarca del Andévalo, así como para la zona limítrofe de la Sierra Norte de Sevilla donde ha sido recolectada en zonas temporalmente inundadas.

Juncus acutiflorus Ehrh. ex Hoffm. subsp. acutiflorus

HUELVA. Almonaster la Real: Embalse del
Toril, orillas, PB9384, 2.6.94, Santa-Bárbara (SEV 151012). Valverde del Camino: Dique de Silillos, QB0168, 22.8.94, Santa-Bárbara (SEV 151013). Villanueva de las Cruces: Río Oraque, PB768671, 19.6.93, Santa-Bárbara (SEV 151014).

Taxón con distribución en Andalucía Occidental restringida a la comarca de Aracena. Recientemente ha sido citado para las comarcas de la Sierra Norte y del Andévalo, en su parte más oriental, perteneciente a la provincia de Sevilla (Pérez Latorre et al., 2002: 204), siendo ésta la segunda cita para la comarca, donde es poco frecuente y se encuentra en herbazales húmedos rodeando zonas encharcadas y cursos de agua.

Juncus acutiflorus subsp. rugosus (Steud.) Cout. HUELVA. Alosno: Entre Alosno y El Almendro, Dehesa Boyal, PB6356, 24.4.96, Santa-Bárbara (SEV 151015). El Cerro del Andévalo: Entre Tharsis y Villanueva de las Cruces, Barranco de la Tiesa, PB6864, 29.5.93, Santa-Bárbara (SEV 151016). Puebla de Guzmán: Rivera Cobica, PB588678, 12.6.93, Santa-Bárbara (SEV 151017). Valverde del Camino: A 2 Km de La Florida, QB0454, 24.5.96, Santa-Bárbara (SEV 151018); ídem, Barranco de la Melera Chica, QB0354, 7.8.96, Santa-Bárbara (SEV 151019). Villanueva de los Castillejos: Huertos del Batán, PB5741, 20.6.96, Santa-Bárbara (SEV 151020). Zalamea la Real: Minas de El Buitrón, PB9668, 22.6.90, Santa-Bárbara (SEV 151021).

Distribuido en las comarcas de Litoral de Huelva y Algeciras. Primera cita para el Andévalo, donde se encuentra en herbazales húmedos y bordes de cursos de agua.

Juncus $\times$ donyanae Fern.-Carv.

HUELVA. Calañas: Río Odiel, PB8252, 7.8.96, Santa-Bárbara (SEV 151055). Zalamea la Real: El Buitrón, camino hacia la mina, PB9768, 22.6.90, Santa-Bárbara (SEV 151056).

Especie endémica de la provincia de Huelva (Fernández Carvajal, 1986: 117) recolectada hasta la fecha únicamente en el Parque Nacional de Doñana. Es la segunda cita de este taxón para la provincia de Huelva y la primera para la comarca del Andévalo. Se ha localizado en ambientes húmedos, en ambos casos en la proximidad de cursos de agua afectados por la actividad minera.

Scirpus maritimus L. subsp. maritimus 
HUElVA. Ayamonte: Arroyo Pedraza, PB4725, 2.7.96, Santa-Bárbara (SEV 151058); ídem, Casa de Matabichos, PB4028, 25.4.97, SantaBárbara \& Valdés (SEV 151059); ídem, Riberas del río Guadiana, PB3925, 25.4.97, Santa-Bárbara \& Valdés (SEV 151060). El Granado: Puerto de la Laja, PB3354, 26.4.97, Santa-Bárbara \& Valdés (SEV 151057). Sanlúcar de Guadiana: Desembocadura de la rivera Grande en el Guadiana, PB3646, 10.10.96, Santa-Bárbara \& Valdés (SEV 151061); ídem, Riberas del río Guadiana, PB3548, 26.4.97, Santa-Bárbara \& Valdés (SEV 151062). Villanueva de los Castillejos: Rivera del Muleto, PB5642, 20.6.96, Santa-Bárbara (SEV 151063).

Frecuente en la Vega, Litoral, Marisma, Campiña Baja, Campiña Alta, Subbética, Grazalema y Algeciras. Recolectado recientemente en la comarca del Andévalo, localizado sólo en el extremo oeste de la comarca. Se encuentra en bordes de ríos, arroyos y charcas.

\section{Scirpus setaceus L.}

HUELVA. Almonaster la Real: Carretera a Valdelamusa, PB9586, 1.5.94, Santa-Bárbara (SEV 151090). Alosno: Entre Villanueva de las Cruces y Tharsis, zona encharcada, PB7263, 15.5.96, SantaBárbara (SEV 151091). Berrocal: Arroyo Las Telliscas, QB 1763, 20.5.90, Arista \& Santa-Bárbara (SEV 151092). El Granado: Cumbre de Pie de Palo, PB4056, 29.5.96, Santa-Bárbara (SEV 151093). Sanlúcar de Guadiana: Entre San Silvestre de Guzmán y Sanlúcar de Guadiana, PB4343, 11.6.94, Santa-Bárbara (SEV 151094).

En Andalucía Occidental este taxón se conocía en Aracena. Es poco frecuente en el Andévalo, donde se cita por primera vez. Se encuentra en zonas encharcadas y bordes de cursos de agua sobre sustrato arenoso.

\section{Scirpus cernuus Vahl}

HUELVA. Almonaster la Real: Sierra Blanca, embalse Olivargas, PB9276, 10.5.90, Santa-Bárbara \& Valdés (SEV 151096); ídem, Embalse del Toril, orillas, PB9384, 2.6.94, Santa-Bárbara (SEV 151097). Alosno: A $5 \mathrm{Km}$ de Alosno hacia el Este, cortijo de la Estación, PB7057, 28.3.94, SantaBárbara \& Valdés (SEV 151098); ídem, entre Alosno y El Almendro, arroyo, PB6256, 24.4.96, Santa-Bárbara (SEV 151099). Ayamonte: Marismas del río Pedrazas, PB4025, 25.4.97, Santa-Bárbara
\& Valdés (SEV 151101). Berrocal: Arroyo Las Telliscas, QB1763, 20.5.90, Arista \& Santa-Bárbara (SEV 151102). Calañas: A $2 \mathrm{Km}$ de Calañas hacia el Cerro del Andévalo, PB87, 16.5.90, Santa-Bárbara \& Valdés (SEV 151103). El Almendro: Barranco del Acebuche, PB3861, 5.5.94, Santa-Bárbara \& Valdés (SEV 151104). El Cerro del Andévalo: Entre Montes de San Benito y Villanueva de las Cruces, depresión con pastizal, PB7069, 15.5.96, SantaBárbara (SEV 151105). El Granado: Carretera al Puerto de la Laja, PB3354, 15.5.93, Santa-Bárbara (SEV 151106). Paymogo: Arroyo que desemboca en el embalse de Paymogo, PB4880, 8.5.96, SantaBárbara (SEV 151107). Puebla de Guzmán: Rivera Malagoncillo, PB6472, 9.4.94, Santa-Bárbara (SEV 151108). Sanlúcar de Guadiana: Entre San Silvestre y Sanlúcar de Guadiana, carril al Guadiana, PB374461, 21.5.94, Santa-Bárbara (SEV 151109); ídem, entre San Silvestre de Guzmán y Sanlúcar de Guadiana, PB4343, 11.6.94, Santa-Bárbara (SEV 151110). Valverde del Camino: Afueras de Valverde, PB9960, 5.4.96, Santa-Bárbara (SEV 151111). Villablanca: Arroyo del Garabato, PB4632, 21.5.94, Santa-Bárbara (SEV 151100); ídem, carril hacia el Guadiana, arroyo Grande, PB4432, 22.5.96, Santa-Bárbara (SEV 151112). Villanueva de los Castillejos: Arroyo de las Multas, PB6549, 26.3.94, Santa-Bárbara (SEV 151113); ídem, entre Villanueva de los Castillejos y San Bartolomé, eucaliptal joven, PB6346, 10.4.96, Santa-Bárbara (SEV 151114); ídem, camino a Casa del Rinconcillo, PB5044, 10.4.96, Santa-Bárbara (SEV 151115); ídem, arroyo del Colmenar, PB4944, 10.4.96, SantaBárbara (SEV 151116).

Distribuida por las comarcas de Aracena, Campiña de Huelva, Litoral, Grazalema y Algeciras, esta especie no había sido citada con anterioridad para el Andévalo, donde presenta una amplia distribución. Se encuentra en suelos arenosos húmedos.

\section{Fuirena pubescens (Poir.) Kunth}

HUELVA. Valverde del Camino: Barranco de la Melera Chica, QB0354, 10.10.96, Santa-Bárbara \& Valdés (SEV 151140).

Tan sólo conocida de las comarcas de Litoral de Huelva y Algeciras. Muy rara en el Andévalo, donde se ha localizado en un único punto en el extremo SE de la comarca, en suelos encharcados sobre sustrato arenoso. 
Fimbristylis bisumbellata (Forssk.) Bubani

HUELVA. Valverde del Camino: Dique de Silillos, QB0168, 22.8.94, Santa-Bárbara (SEV 151141).

Especie citada como localmente frecuente para las comarcas naturales de Sierra Norte de Córdoba, Condado-Aljarafe y Litoral. Muy rara en el Andévalo donde se cita por primera vez. Se ha localizado en bordes de cursos de agua.

\section{Cyperus flavescens $\mathrm{L}$.}

HUELVA. Valverde del Camino: Barranco de la Melera Chica, QB0354, 10.10.96, Santa-Bárbara \& Valdés (SEV 151181).

En lo que a Andalucía Occidental se refiere, este taxón presenta una distribución que comprende las comarcas de los Pedroches, Aracena, Litoral, Subbética, Grazalema y Algeciras. Con esta cita se amplía el área de distribución al Andévalo, donde es rara y sólo se ha localizado en su extremo SE, sobre arenas encharcadas de origen litoral.

\section{Schoenus nigricans L.}

HUELVA. El Almendro: Alrededores del Embalse del Chanza, Era de las Perdices, PB36, 5.5.94, Santa-Bárbara \& Valdés (SEV 151182); ídem, camino a la ermita de Piedras Albas, PB4952, 25.3.95, Santa-Bárbara (SEV 151183). El Cerro del Andévalo: Entre Cabezas Rubias y Calañas, PB7276, 6.5.90, Ferguson, Santa-Bárbara \& Valdés (SEV 151184); ídem, Cumbres de la Hoya, PB88, 16.5.90, Santa-Bárbara \& Valdés (SEV 151185); ídem, entre Cabezas Rubias y Calañas, a $3 \mathrm{Km}$ de Cabezas Rubias, PB7176, 14.5.93, Santa-Bárbara (SEV 151186). Puebla de Guzmán: Carretera a El Almendro, PB5462, 12.6.93, Santa-Bárbara (SEV 151187).

Originalmente citada para las comarcas de Sierra Norte, Litoral, Marisma, Subbética, Grazalema y Algeciras. En el Andévalo, donde se ha recolectado por primera vez, se encuentra en pastizales muy húmedos y bordes de cursos de agua.

\section{Carex flacca subsp. serrulata (Biv.) Greuter}

HUELVA. Calañas: Embalse de Riscoso, PB8563, 4.4.97, Santa-Bárbara \& Valdés (SEV 151214). SEVILLA. El Castillo de las Guardas: Arroyo de Peñas Altas, QB2375, 7.4.93, SantaBárbara (SEV 151215).

Taxón conocido hasta la fecha en las comarcas de Aracena, Litoral, Campiña Alta, Subbética, Grazalema y Algeciras. Se amplía su área de distribución al Andévalo. También ha sido localizado en la Sierra Norte de Sevilla en zonas limítrofes a la comarca. Se encuentra en suelos encharcados de carácter básico. Es rara en el Andévalo.

\section{Carex acuta $\mathrm{L}$.}

HUELVA. Almonaster la Real: Rivera Escalada, PB9887, 1.5.94, Santa-Bárbara (SEV 151217). Nerva: Rivera del Jarama y alrededores, QB2275, 7.4.93, Santa-Bárbara (SEV 151218). Puebla de Guzmán: Río Chanza, PB3773, 26.4.97, Santa-Bárbara \& Valdés (SEV 151219). Rosal de la Frontera: Rivera de Calaboza, PB5899, 13.3.97, Santa-Bárbara \& Valdés (SEV 151220). Valverde del Camino: Arroyo Fresnajoso, PB9163, 4.4.97, Santa-Bárbara \& Valdés (SEV 151221).

Especie distribuida originalmente por las comarcas de Sierra Norte, Aracena, CondadoAljarafe, Vega, Litoral, Grazalema y Algeciras. Ha sido citada recientemente para la comarca del Andévalo, en su extremo oriental (Pérez Latorre $e t$ al., 2002: 204). Con esta cita se amplia su distribución dentro de la comarca, donde se encuentra en bordes de ríos y arroyos.

\section{Festuca arundinacea subsp. atlantigena (St.-Yves) Auquier}

HUELVA. Paymogo: Río Chanza y zonas arenosas contiguas, PB4080 8.5.96, Santa-Bárbara (SEV 151304).

Ampliamente distribuida en Andalucía Occidental, aunque no había sido citada anteriormente en el Andévalo, donde es muy rara. Se ha encontrado en un único enclave, formando parte de un herbazal húmedo.

Festuca ampla Hack. subsp. ampla

HUELVA. Ayamonte: Arroyo Pedraza, PB4725, 15.5.90, Santa-Bárbara (SEV 151296). Berrocal: Arroyo Gallego, QB1866, 5.6.96, SantaBárbara (SEV 151297). El Almendro: Barranco del Acebuche, PB3861, 5.5.94, Santa-Bárbara \& Valdés (SEV 151300). El Cerro del Andévalo: Entre Valdelamusa y San Telmo, Barranco Bartolazo, PB8586, 2.6.94, Santa-Bárbara (SEV 151295); ídem, Cumbres de la Hoya, PB88, 16.5.90, SantaBárbara \& Valdés (SEV 151301). Paymogo: Arroyo 
que desemboca en el embalse de Paymogo, PB479797, 8.5.96, Santa-Bárbara (SEV 151302). Puebla de Guzmán: Márgenes del río Malagón, PB5873, 6.5.90, Ferguson, Santa-Bárbara \& Valdés (SEV 151305). Villanueva de las Cruces: A $2 \mathrm{Km}$ de Villanueva de las Cruces, PB7367, 15.5.96, SantaBárbara (SEV 151306). Carretera antigua entre Zalamea y Valverde, QB0670, 10.5.90, SantaBárbara \& Valdés (SEV 151307). Zalamea la Real: Rivera del Manzano, QB0968, 4.6.90, SantaBárbara (SEV 151308); ídem, Puente de los Cinco Ojos, QB0278, 13.6.90, Santa-Bárbara (SEV 151309), ídem, Rivera de Cachán, QB16, 13.7.90, Santa-Bárbara (SEV 151310).

Citado como muy frecuente en las comarcas de Zújar, Pedroches, Sierra Norte, Aracena, Litoral, Subbética y Grazalema. En el Andévalo ha sido recolectado recientemente en márgenes de arroyos con aguas estacionales.

Vulpia membranacea (L.) Dumort.

HUELVA. Paymogo: Río Chanza y zonas arenosas contiguas, PB4080 8.5.96, Santa-Bárbara (SEV 151408). Valverde del Camino: A $2 \mathrm{Km}$ de La Florida, QB0454, 24.5.96, Santa-Bárbara (SEV 151409).

Esta especie presenta como área de distribución en Andalucía Occidental las comarcas de los Pedroches, Campiña de Huelva, Litoral, Marisma y Campiña Alta de Sevilla. Se amplía su área de distribución a la comarca del Andévalo, donde se ha encontrado en pastizales sobre sustratos arenosos.

\section{Aira caryophyllea L. subsp. caryophyllea}

HUELVA. Alosno: Entre Alosno y El Almendro, Dehesa Boyal, PB6356, 24.4.96, SantaBárbara (SEV 151755). Berrocal: Casa Canijo, QB1662, 20.5.90, Arista \& Santa-Bárbara (SEV 151756). Nerva: Carretera a Sevilla, entrada al Embalse del Jarama, QB2075, 9.4.93, SantaBárbara (SEV 151757). A 1,5 Km de Santa Bárbara, arroyo Albahacar de Allá, PB5885, 19.4.94, SantaBárbara (SEV 151763). Entre Villanueva de los Castillejos y San Bartolomé, eucaliptal joven, PB6346, 10.4.96, Santa-Bárbara (SEV 151758). SEVILLA. El Madroño: A un Km de El Álamo, QB2769, 9.4.93, Santa-Bárbara (SEV 151760).

Citada como rara en Aracena, única comarca en que se había localizado. No muy frecuente en el Andévalo, de donde se cita por primera vez. Se encuentra en pastizales, de espacios abiertos y soleados. También ha sido recolectada en la Sierra Norte de Sevilla, próxima a la comarca del Andévalo.

Aira caryophyllea subsp. uniaristata (Lag. \& Rodr.) Maire

HUELVA. Almonaster la Real: Sierra Blanca, embalse Olivargas, PB9276, 10.5.90, Santa-Bárbara \& Valdés (SEV 151761); ídem, Rivera Escalada, PB9887, 1.5.94, Santa-Bárbara (SEV 151762). Valverde del Camino: Rivera de la Casa de Valverde, QB1057, 30.5.93, Santa-Bárbara (SEV 151764). Carretera antigua entre Zalamea la real y Valverde, QB0670, 10.5.90, Santa-Bárbara \& Valdés (SEV 151759).

Tan sólo localizada en las comarcas de Aracena, Grazalema y Algeciras. Pérez Latorre et al. (2002: 203), la cita para las comarcas de Marisma y Campiña de Huelva. Se ha recolectado en el Andévalo, donde se encuentra en pastizales húmedos sobre sustratos arenosos.

Airopsis tenella (Cav.) Asch. \& Graebn.

HUELVA. Valverde del Camino: Rivera de la Casa de Valverde, QB 1057, 30.5.93, Santa-Bárbara (SEV 151790).

Especie frecuente en Aracena, Litoral, Campiña Baja y Algeciras. Muy rara en la comarca del Andévalo, donde se cita por primera vez.

\section{Corynephorus canescens (L.) P. Beauv.}

HUELVA. Valverde del Camino: A $2 \mathrm{Km}$ de La Florida, QB0454, 5.6.96, Santa-Bárbara (SEV 151827).

Citado como abundante para las comarcas del Litoral y Algeciras. Muy raro en el Andévalo. Localizado en un único enclave al SE de la comarca, sobre arenas de origen litoral.

Corynephorus fasciculatus Boiss. \& Reut.

HUELVA. Ayamonte: Carril entre el río Guadiana y Villablanca, PB3935, 22.5.96, SantaBárbara (SEV 151828). El Almendro: Alrededores del Embalse del Chanza, PB36, 5.5.94, SantaBárbara \& Valdés (SEV 151829). Valverde del Camino: A $2 \mathrm{Km}$ de La Florida, QB0454, 5.6.96, Santa-Bárbara (SEV 151830).

Anteriormente citada para las comarcas de los Pedroches, Sierra Norte, Litoral y Algeciras. Se cita por primera vez para la comarca del Andévalo, 
donde es rara y se encuentra en pastizales sobre suelos arenosos.

Anthoxanthum aristatum Boiss. subsp. aristatum HUELVA. Almonaster la Real: Carretera a Valdelamusa, PB9586, 1.5.94, Santa-Bárbara (SEV 151948). Entre Alosno y El Almendro, Dehesa Boyal, PB6356, 24.4.96, Santa-Bárbara (SEV 151949); ídem, Casas del Picote, PB6255, 24.4.96, Santa-Bárbara (SEV 151950). Calañas: Arroyo del Calabazar, PB883640, 8.4.93, Santa-Bárbara (SEV 151951); ídem, Cabezo entre el apeadero del Cobujón y el río Oraque, PB7956, 2.4.95, SantaBárbara (SEV 151952). El Almendro: Sierra Abuela, PB5552, 4.4.97, Santa-Bárbara \& Valdés (SEV 151953). Entre el Cerro del Andévalo y Valdelamusa, PB87, 16.5.90, Santa-Bárbara \& Valdés (SEV 151954). El Cerro del Andévalo: Entre Montes de San Benito y Villanueva de las Cruces, depresión con pastizal, PB7069, 15.5.96, SantaBárbara (SEV 151955). Entre El Granado y La Isabel, 29.5.96, Santa-Bárbara (SEV 151956). Paymogo: Río Chanza y zonas arenosas contiguas, PB4080 8.5.96, Santa-Bárbara (SEV 151957). Sanlúcar de Guadiana: Rivera Grande, PB3747, 12.3.96, Santa-Bárbara (SEV 151958). Valverde del Camino: Rivera de la Casa de Valverde, QB1057, 30.5.93, Santa-Bárbara (SEV 151959); ídem, entre La Florida y el Poblado del Manzanito, QB0756, 5.4.96, Santa-Bárbara (SEV 151960). Villanueva de las Cruces: Arroyo Monte la Osa, PB7465, 26.2.97, Santa-Bárbara \& Valdés (SEV 151962). Zalamea la Real: Mina de Oriente, QB0466, 29.3.96, Santa-Bárbara (SEV 151963).

A la distribución original, Pedroches, Sierra Norte y Aracena, hay que añadir la comarca del Andévalo donde ha sido localizada recientemente. Se encuentra en pastizales de sitios frescos sobre suelos esqueléticos. Es especialmente frecuente en roquedos.

\section{Phalaris canariensis L.}

HUELVA. Minas de Riotinto: Vertedero de Riotinto, QB1474, 7.4.93, Santa-Bárbara (SEV 151965). Valverde del Camino: Entre Valverde y Poblado del Manzanito, QB05, 30.5.93, SantaBárbara (SEV 151966).

De distribución amplia en Andalucía Occidental, no había sido citada con anterioridad para la comarca del Andévalo. Es muy rara en esta comarca, encontrándose en enclaves ruderalizados.

\section{Glyceria declinata Bréb.}

HUELVA. Alosno: Entre Villanueva de las Cruces y Tharsis, zona encharcada, PB7263, 15.5.96, Santa-Bárbara (SEV 151999). Calañas: Entre el apeadero de la Encarnación y el apeadero del Cobujón, PB8156, 2.4.95, Santa-Bárbara (SEV 152000). Villanueva de las Cruces: A un Km del pueblo, arroyo Chapinero, PB752670, 19.6.93, Santa-Bárbara (SEV 152001).

Esta especie fue citada originalmente como frecuente para las comarcas de Zújar, Sierra Norte, Aracena, Litoral, Grazalema y Algeciras. Rara en la comarca del Andévalo, de donde no se había citado anteriormente. Se encuentra en bordes de charcas y lugares encharcados.

\section{Bromus intermedius Guss.}

HUELVA. Valverde del Camino: Rivera de la Casa de Valverde, QB 1057, 30.5.93, Santa-Bárbara (SEV 152015). Zalamea la Real: Barranco de las Rebujas, QB1663, 5.6.96, Santa-Bárbara (SEV 152016).

Distribuida por las comarcas de Sierra Norte, Aracena, Subbética y Grazalema. No citada anteriormente para el Andévalo, donde es muy rara. Ha sido localizada solamente en su extremo suroriental, en pastizales de lugares frescos con sustratos básicos.

Brachypodium sylvaticum (Huds.) P. Beauv.

HUELVA. Nerva: Barranco de los Cuarteles, QB1378, 3.7.90, Santa-Bárbara (SEV 152145). Zalamea la Real: Barranco de las Rebujas, QB 1663, 5.6.96, Santa-Bárbara (SEV 152146).

Se encuentra en barrancos umbríos y húmedos siendo muy rara en la comarca. Es de destacar que el material de las dos localidades del Andévalo presenta hojas y glumas glabras o subglabras, caracteres que según Talavera (1987: 369) separan B. sylvaticum (Huds.) P. Beauv. de B. gaditana Talavera. Por su parte, Schippmann (1991: 166) incluye $B$. gaditanum Talavera como sinónimo de B. sylvaticum (Hudson) Beauv. Al margen de considerar o no como taxón independiente a $B$. gaditanum Talavera, las medidas de los caracteres florales de los ejemplares localizados en el Andévalo se corresponden con los de B. sylvaticum (Huds.) P. Beauv., de manera que serían formas subglabras de 
esta especie.

\section{Hordeum geniculatum All.}

HUELVA. Sanlúcar de Guadiana: Riberas del río Guadiana, PB3548, 26.4.97, Santa-Bárbara \& Valdés (SEV 152234).

Presenta su área de distribución en las comarcas de los Pedroches, Litoral, Marisma, Subbética de Córdoba, Grazalema y Algeciras. Muy rara en el Andévalo, comarca de donde se cita por primera vez. Se ha localizado en su extremo occidental, en terrenos con encharcamiento periódico.

\section{Stipa gigantea Link}

HUELVA. El Almendro: Sierra Abuela, PB5552, 4.4.97, Santa-Bárbara \& Valdés (SEV 152276). Nerva: Alrededores del embalse del Jarama, QB2276, 7.4.93, Santa-Bárbara (SEV 152277).

Citada para las comarcas de Aracena, Litoral, Alcores, Campiña Baja, Campiña Alta, Subbética, Grazalema y Algeciras. En el Andévalo, donde se cita por primera vez, se encuentra sobre sustratos arenosos en suelos secos y soleados.

Crypsis schoenoides (L.) Lam.

HUELVA. Valverde del Camino: Arroyo Bajohondo, QB0061, 22.8.94, Santa-Bárbara (SEV 151990).

Castroviejo et al. (1980: 233) citan esta especie para el Parque Nacional de Doñana, Litoral de Huelva. Posteriormente el área de distribución en Andalucía Occidental quedó establecido en las comarcas de los Pedroches, Sierra Norte, CondadoAljarafe, Vega, Litoral gaditano, Campiña Baja y Campiña Alta. Recientemente ha sido localizada en el Andévalo, donde es muy rara. Se ha recolectado en un pastizal de un terreno con encharcamiento temporal.

Paspalum dilatatum Poir.

HUELVA. El Granado: Puerto de la Laja, PB3354, 10.10.96, Santa-Bárbara \& Valdés (SEV 152311). Sanlúcar de Guadiana: Riberas del Guadiana, PB3548, 30.9.97, Santa-Bárbara (SEV 152310).

Especie oriunda de America del sur. En Andalucía Occidental ha sido citada en las comarcas de Aracena y Algeciras. Sánchez García \& Martínez
Ortega (1994: 365) amplían el área de distribución a la Campiña Baja de Sevilla. Primera cita para el Andévalo, donde ha sido localizada tan sólo en el extremo occidental de la comarca, creciendo en suelos encharcados o muy húmedos.

Phragmites australis (Cav.) Trin. ex Steud. subsp. australis

HUELVA. Ayamonte: Barranco de la Chacera, PB3936, 22.5.96, Santa-Bárbara (SEV 152341). El Granado: Huerta de las Palomas, río Guadiana, PB3352, 21.11.96, Santa-Bárbara \& Valdés (SEV 152340); ídem, Rivera de la Rochona, PB3550, 21.11.96, Santa-Bárbara \& Valdés (SEV 152338). Minas de Riotinto: Carretera a Monte Sorromero, QB1372, 12.8.94, Santa-Bárbara (SEV 152343). Sanlúcar de Guadiana: Entre San Silvestre de Guzmán y Sanlúcar de Guadiana, PB4045, 11.6.94, Santa-Bárbara (SEV 152346); ídem, desembocadura de la rivera Grande en el Guadiana, PB3646, 10.10.96, Santa-Bárbara \& Valdés (SEV 152339).

Taxón citado para las comarcas de Litoral, Subbética, Grazalema y Algeciras. Localizado en el extremo occidental del Andévalo, en las orillas de las riveras que vierten al río Guadiana.

\section{Arundo plinii Turra}

HUELVA. El Granado: Río Guadiana, PB3156, 26.4.97, Santa-Bárbara \& Valdés (SEV 152348).

La primera cita para Andalucía Occidental de esta especie, la situa en el Litoral de Huelva, dentro del Parque Nacional de Doñana (Cabezudo, 1979: 179). Posteriormente se ha indicado en las comarcas de la Vega, Marisma y Subbética de Cádiz. Con esta cita se amplía su área de distribución al Andévalo, donde es muy rara. Se encuentra en los sotos de los márgenes del río Guadiana, en enclaves poco alterados.

Molinia caerulea subsp. arundinacea (Schrank) K. Richt.

HUELVA. Zalamea la Real: Puente de los Cinco Ojos, QB0278, 13.6.90, Santa-Bárbara (SEV 152349).

Taxón frecuente en la Campiña de Huelva, Condado-Aljarafe, Litoral, Grazalema y Algeciras. Pérez Latorre et al. (2002: 204) amplía su área de distribución a la comarca del Andévalo, a su extremo oriental dentro de la provincia de Sevilla. Constituye ésta la segunda cita para el Andévalo donde es muy 
rara y se ha localizado en un único enclave, en un pastizal aledaño al curso alto del río Odiel al $\mathrm{N}$ de la comarca.

Sparganium erectum L. subsp. erectum

HUELVA. San Bartolomé de la Torre: Arroyo y embalse de San Bartolomé, PB6647, 20.6.96, Santa-Bárbara (SEV 152350).

Distribución limitada a las comarcas de Litoral de Huelva y Marisma. Muy rara en el Andévalo, donde se ha recolectado por primera vez en un único punto, en el borde de un embalse, en una zona muy degradada.

\section{Ornithogalum pyrenaicum $\mathrm{L}$.}

HUELVA. El Granado: Carretera al río Guadiana, cruce, PB3853, 15.5.93, Santa-Bárbara (SEV 152413). Puebla de Guzmán: Márgenes del río Malagón, PB5873, 6.5.90, Ferguson, SantaBárbara \& Valdés (SEV 152414). Villanueva de las Cruces: A $2 \mathrm{Km}$ de Villanueva de las Cruces, PB7367, 15.5.96, Santa-Bárbara (SEV 152415).

Especie rara con distribución restringida a la Sierra Norte de Córdoba. Poco frecuente en la comarca del Andévalo, donde se cita por primera vez. Se encuentra entre el matorral en sotos de ríos $\mathrm{y}$ arroyos.

\section{Allium neapolitanum Cirillo}

HUELVA. Villanueva de los Castillejos: Arroyo del Colmenar, PB4944, 10.4.96, SantaBárbara (SEV 142888).

Ampliamente distribuida en Andalucía Occidental, no había sido citada con anterioridad para la comarca del Andévalo, donde es muy rara. Se ha localizado en las proximidades de un curso de agua.

\section{Allium massaessyllum Batt. \& Trab.}

HUELVA. Almonaster la Real: Rivera Escalada, PB9887, 1.5.94, Santa-Bárbara (SEV 142889). Calañas: Embalse de Riscoso, PB8563, 4.4.97, Santa-Bárbara \& Valdés (SEV 142964). La Puebla de Guzmán: Rivera Malagoncillo, PB6472, 9.4.94, Santa-Bárbara (SEV 145424). Entre Santa Bárbara de Casas y Paymogo, barranco de Monteviejo, PB5482, 19.4.94, Santa-Bárbara (SEV 142980). Valverde del Camino: Barranco de la mina de la Cara, QB103572, 30.5.93, Santa-Bárbara (SEV 143044); ídem, Rivera de la Casa de Valverde,
QB1057, 5.4.96, Santa-Bárbara (SEV 143083).

Presenta como área de distribución en Andalucía Occidental las comarcas de Sierra Norte, Aracena y Campiña de Huelva. Ha sido recolectada recientemente en el Andévalo, donde es poco frecuente, encontrándose en pastizales y herbazales húmedos.

\section{Asparagus albus L.}

HUELVA. Ayamonte: Huertos que rodean la margen izquierda del río Guadiana, PB3836, 22.5.96, Santa-Bárbara (SEV 144680); ídem, Riberas del río Guadiana, PB3925, 25.4.97, Santa-Bárbara \& Valdés (SEV 144704). Cabezas Rubias: Rivera Charcalino, PB6978, 9.4.94, Santa-Bárbara (SEV 144816). Calañas: Río Oraque, paredones rocosos de la orilla izquierda del río, PB7855, 2.4.95, SantaBárbara (SEV 142452). Cerca de El Cerro del Andévalo, PB8278, 3.12.95, Santa-Bárbara (SEV 142471). Puebla de Guzmán: Ermita de la Virgen de la Peña, PB5863, 25.2.95, Santa-Bárbara (SEV 142472). Sanlúcar de Guadiana: El Romerano, roquedo en las proximidades del río Guadiana, PB3641, 13.12.97, Santa-Bárbara (SEV 144319). A aproximadamente $2 \mathrm{Km}$ de Villablanca, PB470264, 25.2.94, Santa-Bárbara \& Valdés (SEV 142643).

Aunque este taxón presenta una amplia distribución en Andalucía Occidental, no había sido citado con anterioridad para la comarca del Andévalo, donde crece en matorrales sobre suelos con cierta concentración de bases. Es más frecuente en roquedos.

Narcissus bulbocodium subsp. obesus (Salisb.) Maire

HUELVA. Valverde del Camino: Cruce entre la carretera de Calañas y la N-435, PB9455, 24.3.96, Santa-Bárbara (SEV 144045).

Únicamente citado para las comarcas de Litoral y Algeciras. Con esta cita se amplía su área de distribución al Andévalo, donde es muy rara. Se ha localizado exclusivamente al S del territorio, ligada al material de construcción de una vía por lo que se le supone un origen alóctono.

\section{Iris albicans Lange}

HUELVA. Valverde del Camino: Entre Los Pinos y Embalse de Campanario, PB95, 18.4.90, Santa-Bárbara \& Valdés (SEV 145758). 
Especie procedente de Arabia ampliamente cultivada en la región mediterránea y naturalizada en las comarcas de Aracena, Litoral y Algeciras. En el Andévalo no se había localizado hasta el momento encontrándose entre el matorral del borde de una vía.

\section{Freesia refracta (Jacq.) Eckl. ex Klatt}

HUELVA. Almonaster la Real: Entre mina Concepción y San Platón, QB0482, 14.3.90, SantaBárbara (SEV 150593).

En Andalucía Occidental se conocía naturalizada en el Litoral y áreas costeras de Algeciras. En el Andévalo ha sido localizada en un único punto, al $\mathrm{N}$ del territorio en los jardines abandonados de unas ruinas.

\section{Orchis lactea Poir.}

HUELVA. Calañas: Sotiel Coronada, PB8866, 20.3.93, Santa-Bárbara (SEV 147317).

Especie frecuente en las comarcas de los Pedroches, Sierra Norte, Aracena, Campiña Alta, Subbética, Grazalema y Algeciras. Muy rara en el Andévalo de donde no se conocía hasta el momento. Se ha localizado entre la vegetación arbustiva pionera tras un incendio, en una ladera de solana.

\section{Orchis laxiflora Lam. subsp. laxiflora}

HUELVA. Alosno: Entre Alosno y El Almendro, arroyo, PB6256, 24.4.96, Santa-Bárbara (SEV 147378).

Citada como frecuente en la Sierra Norte, Litoral, Campiña Alta, Subbética, Grazalema y Algeciras. Muy rara en la comarca del Andévalo, donde se ha encontrado por primera vez en un pastizal húmedo.

\section{BIBLIOGRAFÍA}

CABEZUDO, B. -1979- Plantas de la Reserva Biológica de Doñana (Huelva). II. Lagascalia 8: 167-181.

CASTROVIEJO, S., E. VALDÉS-BERMEJO, S. RIVAS-MARTÍNEZ y M. COSTA - 1980 Novedades florísticas de Doñana. Anales Jardín Bot. Madrid 36: 203-244.

FERNÁNDEZ CARVAJAL, M. C. -1986- Juncus x donyanae Ferández-Carvajal, hybr. novus. Lagascalia 14(1): 117.
FERNÁNDEZ GALIANO, E. y B. CABEZUDO 1976- Plantas de la Reserva Biológica de Doñana (Huelva). Lagascalia 6: 117-176.

PÉREZ LATORRE, A. V., P. NAVAS, D. NAVAS, Y. GIL y B. CABEZUDO -2002- Datos sobre la flora y vegetación de la cuenca del Río Guadiamar (Sevilla-Huelva, España). Acta Bot. Malacitana 27: 189-228.

SÁNCHEZ GARCÍA, I. y C. MARTÍNEZ ORTEGA -1994- Notas taxonómicas y corológicas para la flora vascular de Andalucía y del Rif. Nota 30. Lagascalia 12(2): 357-366.

SANTA-BÁRBARA，C. - 1997 - Novedades corológicas para la comarca del Andévalo (Huelva). I. Lagascalia 20: 170-184.

SANTA-BÁRBARA, C. -1997b- Novedades corológicas para la comarca del Andévalo (Huelva). II. Acta Bot. Malacitana 22: 243.

SANTA-BÁRBARA, C., B. VALDÉS y F. J. PINA -2003- Novedades corológicas para la comarca del Andévalo (Provincia de Huelva, SO de España). III. Lagascalia. En prensa.

SCHIPPMANN, V. -1991-Revision der europäischen Arten der Gattung Brachypodium Palisot de Beauvois (Poaceae). Boissiera 45: 1 250.

TALAVERA, S. -1987- Brachypodium Beauv., en B. Valdés, S. Talavera \& E. Fernández Galiano (eds.). Flora Vascular de Andalucía Occidental 3: 369-372. Ketres Editora S.A., Barcelona.

VALDÉS. B., S. TALAVERA y E. FERNÁNDEZ GALIANO (eds.) -1987- Flora Vascular de Andalucía Occidental 1-3. Ketres Editora S.A., Barcelona.

Aceptado para su publicación en marzo de 2003

Dirección de los autores. Dpto. Biología Vegetal y Ecología, Universidad de Sevilla. Apdo. 1095, E41080 Sevilla 